\title{
Pleomorphic lobular carcinoma in situ: Current evidence and a systemic review (Review)
}

\author{
UMAR WAZIR $^{1}$, ALI WAZIR $^{2}$, CLIVE WELLS $^{1}$ and KEFAH MOKBEL ${ }^{1}$ \\ ${ }^{1}$ Department of Oncoplastic Surgery and Surgical Oncology, The London Breast Institute, Princess Grace Hospital, London, UK; \\ ${ }^{2}$ Department of Internal Medicine, Albany Medical Center, Albany, NY, USA
}

Received July 19, 2015; Accepted April 29, 2016

DOI: $10.3892 / \mathrm{ol} .2016 .5331$

\begin{abstract}
Pleomorphic lobular carcinoma in situ (PLCIS) has only recently been identified as a distinct pathological entity within classic lobular carcinoma in situ (CLCIS). As such, there is currently no consensus among clinicians regarding the optimal treatment of this disease. The present study determined the risk of concomitant invasive disease and ductal carcinoma in situ (DCIS) if PLCIS is observed on core needle biopsy $(\mathrm{CNB})$ and collated the evidence regarding the risk of recurrence in relation to surgical margins and adjuvant therapy. In addition, the pertinent literature available through MedLine, PubMed, the WHO Clinical Trials Registry Platform and Google Scholar using appropriate keywords was reviewed. The pooled results of studies in the literature demonstrated a concomitant presence of invasive disease of $40 \%$, and $15 \%$ for DCIS. The studies that examined recurrence rates indicated that the risk is reduced with ample resection margins (>2 mm) and adjuvant radiotherapy. However, recent studies raise concerns regarding breast conservation when pursuing clear margins. No level 1 evidence from prospective studies, randomized controlled trials (RCTs), or meta-analyses based on such RCTs was identified. This is a clinical issue that warrants investigation in appropriately powered well designed prospective studies for a satisfactory resolution of all concerns.
\end{abstract}

\section{Contents}

1. Background

2. CLCIS

3. PLCIS (Figs. 1-5)

4. The incidence of concomitant invasive disease with an initial diagnosis of PLCIS

Correspondence to: Professor Kefah Mokbel, Department of Oncoplastic Surgery and Surgical Oncology, The London Breast Institute, Princess Grace Hospital, 45 Nottingham Place, London W1U 5NY, UK

E-mail: kefahmokbel@hotmail.com

Key words: breast cancer, lobular carcinoma in situ, surgical excision, pleomorphic
5. The effect of surgical margins and adjuvant therapy on recurrence rates

6. Conclusion

\section{Background}

Pleomorphic lobular carcinoma in situ (PLCIS) has only recently been identified as a distinct pathological entity. The first mention of this lesion was in an unindexed case report by Frost et al (1) in 1996. It has been characterised as an aggressive subtype of classic lobular carcinoma in situ (CLCIS). However there is yet to be a consensus among clinicians regarding the optimal treatment of this disease. As recently as 2013 a survey presented in the San Antonio Breast Cancer Symposium demonstrated that there were significant differences in opinion among breast surgeons regarding the optimal excision margins required to adequately treat PLCIS to prevent recurrences in the future (2).

In this article, the literature available with regards to this disease were reviewed, including the evidence that distinguishes it from other neoplasms of the breast and the results of the significant clinical studies which have looked into this disease were collated and summarised. Specifically, the risk of concomitant invasive disease or ductal carcinoma in situ (DCIS) if PLCIS is found on core needle biopsy (CNB) was determined and the evidence regarding the risk of recurrence in relation to surgical margins and adjuvant therapy was collated.

For the purposes of this systematic review, MedLine, PubMed, the WHO International Clinical Trial Registry Platform and Google Scholar were searched using the following keywords: 'pleomorphic lobular carcinoma in situ', 'pleomorphic lobular carcinoma in situ' and 'PLCIS'. Further articles were identified by manual search through the references in previous reviews and studies $(3,4)$.

\section{CLCIS}

CLCIS has long been associated with an increased risk of carcinoma. However, in a seminal study in 1978, it was observed that the disease was frequently bilateral and too extensive for surgical excision. Also, it was noted that the invasive disease associated with CLCIS was often invasive ductal carcinoma 
(IDC) rather than lobular carcinoma (5). On the basis of these observations, CLCIS is regarded to be a marker of increased risk of invasive disease rather than a precursor of invasive disease (6). It is associated with a risk of invasive breast cancer over 10 years of at least $7.1 \%$. Currently, patients with CLCIS are offered surveillance with regular physical examination and imaging rather than surgical excision (7).

Notably, a recent study suggests that in some instances, CLCIS cells may be clonally related to subsequent invasive lobular carcinoma (ILC), which may suggest that CLCIS may serve as a precursor to invasive disease (8).

\section{PLCIS}

PLCIS was initially described by Frost et al (1), who described the pathology in the context of a 78 years old woman with an ill-defined mass in the right breast and corresponding calcification on a recent mammogram. The lesion was identified by needle localisation and a biopsy was performed followed by wide local excision and radiotherapy. The lesion was grossly characterised as 'ill defined, multifocal and difficult to measure'. Frost et al (1) described the microscopic appearance of the cells as large cells with abundant eosinophilic cytoplasm with irregular nuclei with prominent single or multiple nucleoli. The cells extended into the ducts in a pagetoid fashion and filled the duct lumen with tightly packed cells that lacked cohesion. On immunohistochemistry the cells tested positive for gross cystic disease fluid protein 15 (GCDFP 15) and the c-erB-2 oncogene (1). PLCIS has also been referred to as 'florid' lobular carcinoma in situ, and is clinically and radiologically similar to ductal carcinoma in situ (9).

In the original description by Frost et al, PLCIS was characterised as a hormone receptor negative lesion, in which oestrogen and progesterone receptors were not detected (ER-/PR-) (1), as illustrated in Fig. 1. Since then it has been determined that PLCIS is often a strongly Her2 positive lesion (Her2+), as illustrated in Fig. 2. An additional recent study noted significant Her 2 overexpression and amplification and speculated that this may be involved in the pathogenesis of PLCIS (10). This is in contrast to CLCIS, which is commonly a hormone receptor positive and Her2 negative lesion (ER+/PR+/Her2-) $(11,12)$. Androgen receptor expression is similar in both PLCIS and CLCIS (13). These distinctions have profound implications in terms of prognosis and significantly limit the potential options available for the adjuvant therapy of PLCIS.

It is well recognised that the differentiation of PLCIS from DCIS, on cytological findings alone may be difficult. It has been found that in common with CLCIS and in contrast to DCIS, PLCIS is characterised by a loss of heterozygosity for the E-Cadherin gene, as illustrated in Fig. 3. This is a characteristic it shares with CLCIS which may indicate a clonal relationship (14).

However, it has long been noted that unlike CLCIS, PLCIS may be frequently associated with invasive disease and has more aggressive course $(4,9)$. PLCIS is considered to be a precursor of invasive disease rather than a marker of increased risk like CLCIS. This is borne out by similarities in biomarkers of synchronous PLCIS and pleomorphic invasive lobular carcinoma (PILC). PLCIS is characterised

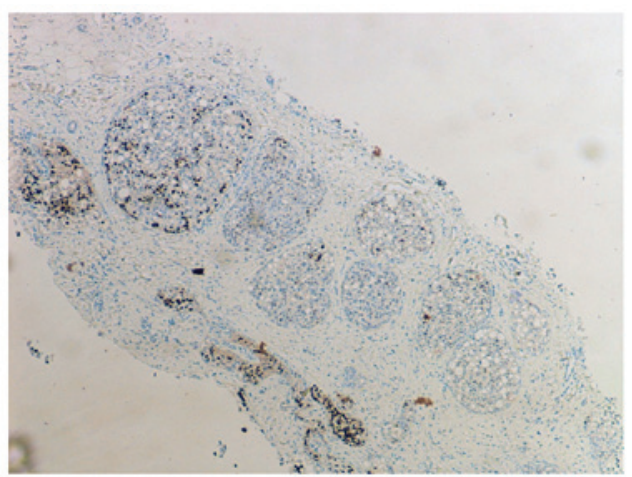

Figure 1. Estrogen receptor staining in PLCIS. Immunohistochemistry for oestrogen receptor showing weak and variable oestrogen receptor staining in a sample of breast tissue with PLCIS at $\mathrm{x} 40$ magnification. Staining was performed using ER/PR pharmdx kits supplied by Dako UK Ltd, (Ely, UK). PLCIS, pleomorphic lobular carcinoma in situ.

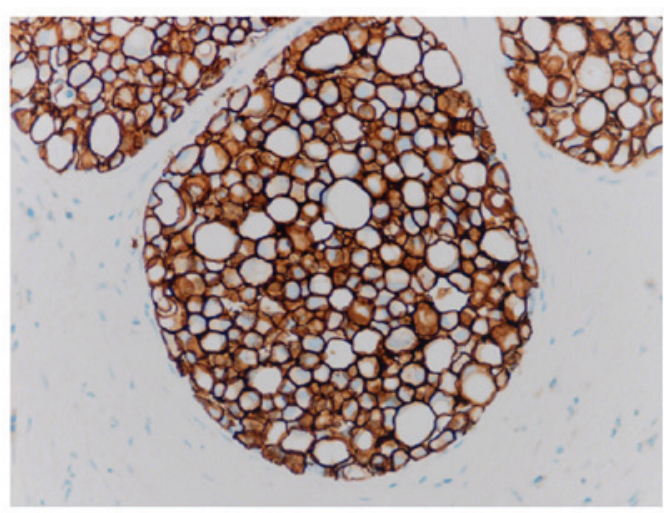

Figure 2. Her2 staining in PLCIS. Immunohistochemistry for Her2 showing strong positivity in a sample of breast tissue with PLCIS at x200 magnification. PLCIS, pleomorphic lobular carcinoma in situ.

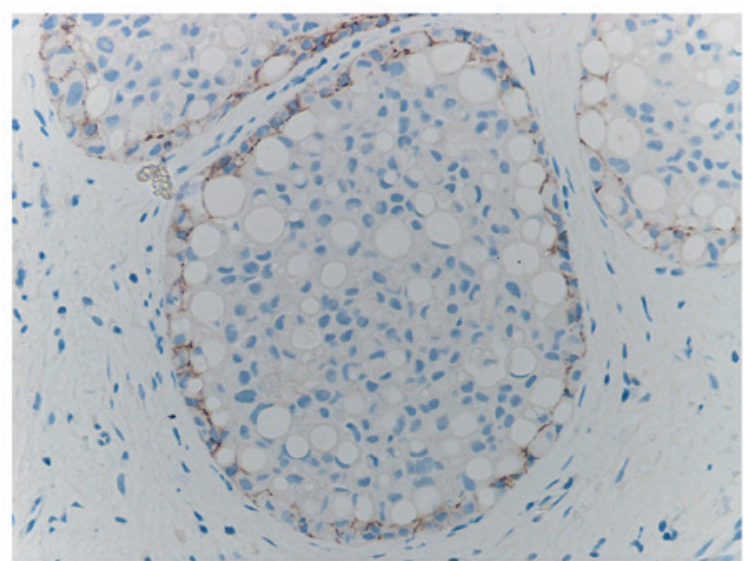

Figure 3. E-cadherin staining in PLCIS. Immunohistochemistry showing loss of e-cadherin staining in a sample of breast tissue with PLCIS at x200 magnification. PLCIS, pleomorphic lobular carcinoma in situ.

by increased expression of Ki67 compared to CLCIS, which indicates a high cell turnover. These observations suggest that this may be a radiosensitive lesion unlike LCIS (12).

This early characterisation of PLCIS as a precursor to invasion more akin to DCIS, however, was based on impressions gleaned from several histological series earlier in the 


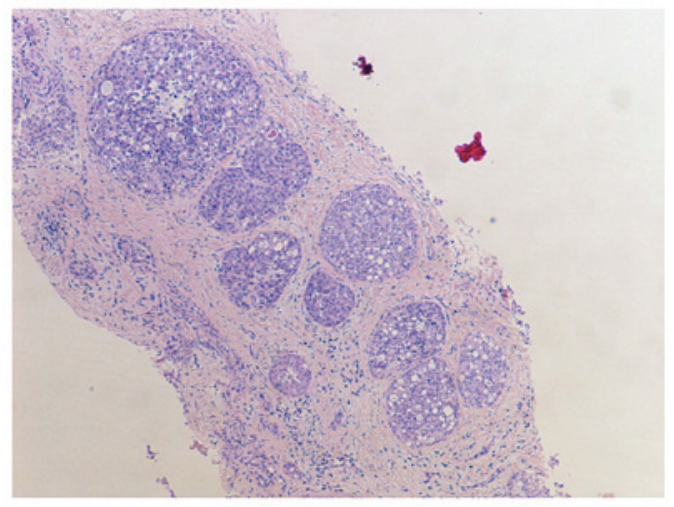

Figure 4. Core biopsy showing expanded acini filled with a pleomorphic proliferation of cells. Haematoxylin and eosin, x40 magnification.

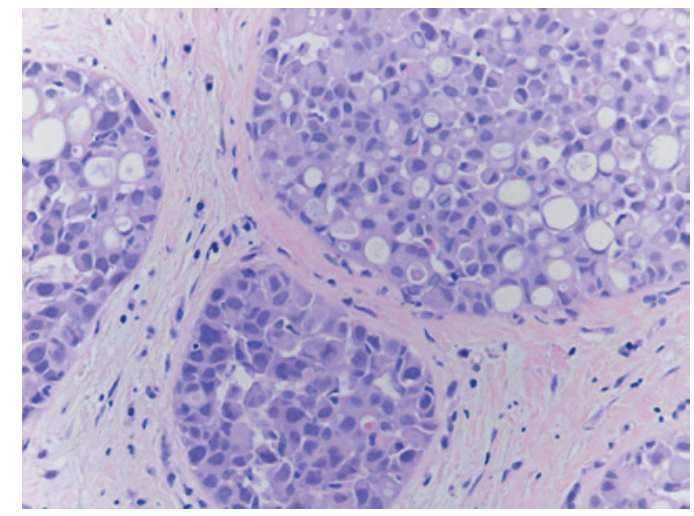

Figure 5. Higher power showing the pleomorphism of the cells but also lobular features with intracytoplasmic lumina. Haematoxylin and eosin, x200 magnification.

previous decade $(15,16)$. Data, albeit retrospective, regarding actual recurrence rates after excision and the presence of concomitant invasive disease have only recently become available. This evidence is reviewed in the following sections.

\section{The incidence of concomitant invasive disease with an initial diagnosis of PLCIS}

Following the search of the aforementioned medical databases and perusing the citations used in previous articles, 10 case series were identified in which the diagnosis of PLCIS was made on CNB. A total of 121 such patients were recorded in these 10 studies (Table I) (17-25).

While case reports were excluded, any patients who formed a part of a larger series in view of the rarity of this condition were included. In all these cases, the patients underwent a core needle biopsy that returned a diagnosis of PLCIS, either alone or in conjunction with another finding such as DCIS, CLCIS or invasion. Furthermore, these patients underwent surgical excision and the authors of these studies reported the final pathological diagnosis.

Of these 121 patients, 81 were recorded to have PLCIS alone on CNB. Of the remainder, 30 exhibited invasion or microinvasion and 7 possessed DCIS. A total of 93 of the patients had wide local excisions (WLE), 3 had diagnostic excisional biopsies and 23 patients had mastectomies.
After final histopathology of the surgical specimen only $33 \%(n=40)$ of the total were determined to not have concurrent disease. Of the remainder, $40.5 \%$ of the cases reported some form of invasive disease, predominantly invasive lobular carcinoma. Other invasive carcinomas reported were invasive ductal carcinoma and additional instances of lobular microinvasion. In addition, $16 \%(\mathrm{n}=19)$ of the cases were reported to have concurrent DCIS. These results are in keeping with previous results from case series and systematic reviews. However, it must be noted that all the data in question was collected retrospectively and would be prone to the biases and confounding factors inherent to such data.

\section{The effect of surgical margins and adjuvant therapy on recurrence rates}

The question of the risk of recurrence following excision of PLCIS is understandably a question of great interest to clinicians. It has long been understood that the clinical history of PLCIS is distinct from that of CLCIS, with behaviour more consistent with a precursor of invasive disease rather than a marker of increased risk. The present review of the literature since the identification of PLCIS as a distinct pathological diagnosis identified four studies that comment upon excision margins and recurrence of the disease (Table II).

The first study reported in the literature is that of Downs-Kelly et al (26) in 2011, which reported on 26 patients who underwent resection for PLCIS and also received adjuvant chemo and radiotherapy. These patients were identified retrospectively from the hospital database. Of these, 20 had PLCIS on pathology, a further 6 patients with an invasive component were included if they were classified as suspicious for microinvasion or invasion measuring between $1-5 \mathrm{~cm}$ and $>1 \mathrm{~cm}$ from the resection margins. The basis for these criteria is unclear. Furthermore, the patients were stratified according to their resection margins. Of these patients, 10 patients did not receive any adjuvant therapy, 7 received chemotherapy only and 3 received radiotherapy only. The remainder received both chemo- and radiotherapy. Again, the basis for this was not clarified in the text by the authors. This group of patients was followed up for a mean of 46 months. In this time, only one recurrence was reported at 19 months, which was PLCIS (3.8\%). The authors remark that the patient in question had disease at the margin and had only received chemotherapy. On the basis of this data the authors recommend treating PLCIS as one would treat DCIS with excision margins of at least $2 \mathrm{~mm}$ and to consider adjuvant therapy (26).

The second study in question is that of Fasola et al (19) of which only an abstract is publically available. Out of 34 patients, all of whom underwent wide local excision, the authors report three local recurrences over a 5-year period. In their cohort, the authors noted PLCIS alone had a higher local recurrence rate compared to PLCIS associated with invasion. It was also noted that the cases which developed local recurrences following excision of PLCIS alone did not receive surgical re-excision or adjuvant therapy and the surgical margins were within $1 \mathrm{~mm}$ of the lesion (19).

Khoury et al (27) report on a case series of 47 patients diagnosed with PLCIS followed up over a 12-year period. Within this group, the authors selected 31 patients with PCLIS in order 


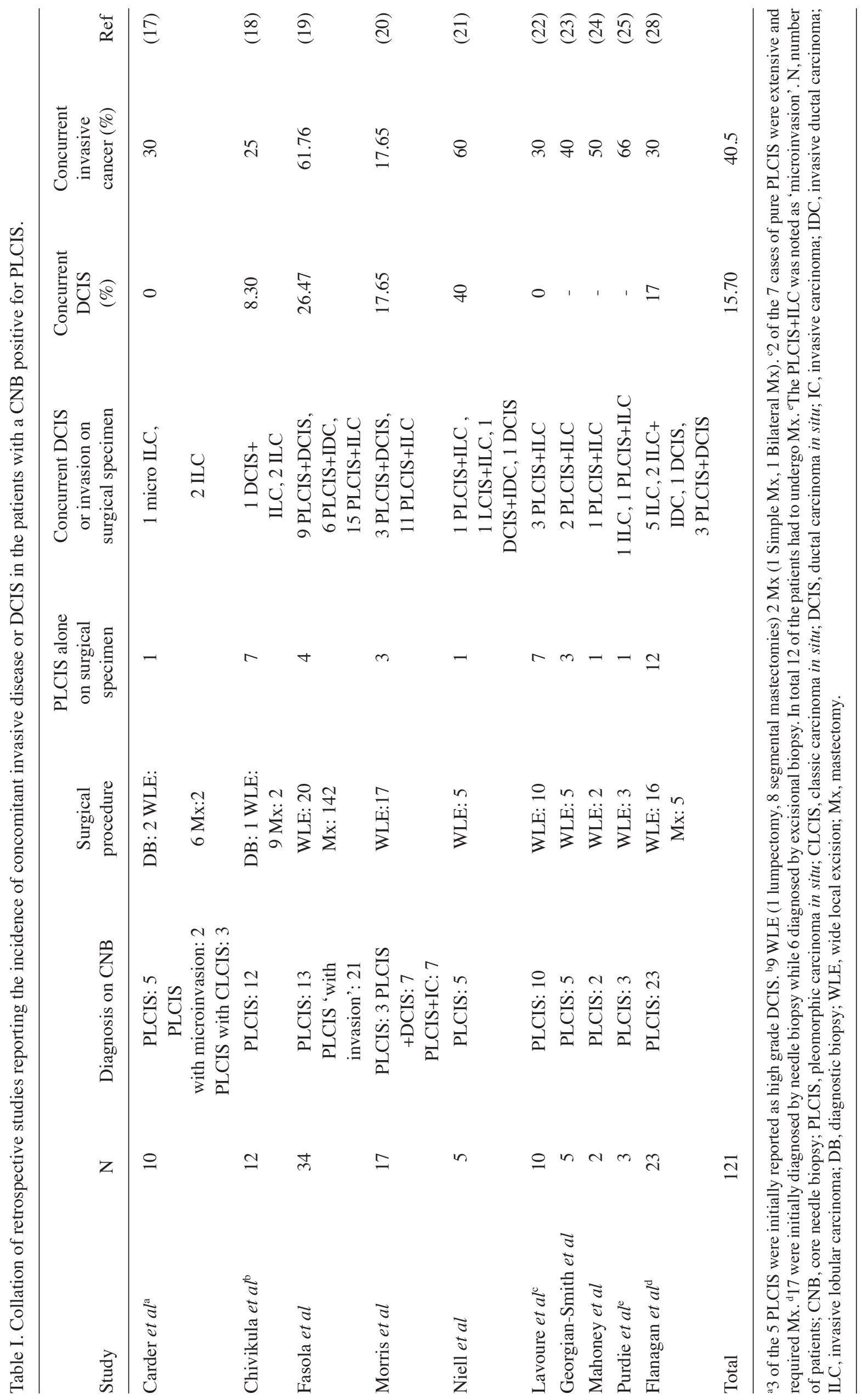


Table II. Summary of retrospective studies reporting local recurrence rates after excision of PLCIS.

\begin{tabular}{|c|c|c|c|c|c|c|c|c|c|}
\hline Study & $\begin{array}{l}\text { Median } \\
\text { follow-up } \\
\text { (months) }\end{array}$ & $\begin{array}{l}\text { Median } \\
\text { age }\end{array}$ & $\mathrm{N}$ & $\begin{array}{l}\text { Surgical } \\
\text { margins }\end{array}$ & $\begin{array}{l}\text { Adjuvant } \\
\text { therapy }\end{array}$ & $\begin{array}{l}\text { Histology } \\
\text { of } \\
\text { recurrences }\end{array}$ & $\begin{array}{l}\text { Time to } \\
\text { recurrence } \\
\text { (months) }\end{array}$ & LRR (\%) & Ref \\
\hline $\begin{array}{l}\text { Fasola } \\
\text { et al }\end{array}$ & $\begin{array}{c}57 \\
(12-163)\end{array}$ & $\begin{array}{c}55 \\
(41-84)\end{array}$ & 34 & $\leq 1 \mathrm{~mm}: 34$ & $\begin{array}{l}\mathrm{RT}+/- \\
\mathrm{CT}: 16 \\
\mathrm{HT}: 9^{\mathrm{c}}\end{array}$ & $\begin{array}{c}\text { Not } \\
\text { stated }\end{array}$ & $\begin{array}{c}\text { Not } \\
\text { stated }\end{array}$ & 8.82 & (19) \\
\hline $\begin{array}{l}\text { Downs } \\
\text {-Kelly } \\
\text { et al }\end{array}$ & $\begin{array}{c}32.5 \\
(4-108)\end{array}$ & $\begin{array}{c}59.5 \\
(35-76)\end{array}$ & 26 & $\begin{array}{l}\text { At margin: } 6 \\
\quad<1 \mathrm{~mm}: 7 \\
1.1-2 \mathrm{~mm}: 4 \\
>2.1 \mathrm{~mm}: 9\end{array}$ & $\begin{array}{c}\text { No Rx: } 10 \\
\text { CRT: } 6 \\
\text { CT: } 6 \\
\text { RT: } 4\end{array}$ & PLCIS: 1 & 18 & 3.84 & (26) \\
\hline $\begin{array}{l}\text { Khoury } \\
\text { et } a l^{\mathrm{a}}\end{array}$ & $\begin{array}{c}55.6 \\
(1.6-112)\end{array}$ & $\begin{array}{c}59.5 \\
(40-88)\end{array}$ & 31 & Not stated & $\begin{array}{c}\text { RT: } 3 \\
\text { HT: } 11\end{array}$ & $\begin{array}{c}\text { PLCIS: } 2 \\
\text { Invasion: } 4\end{array}$ & $\begin{array}{c}\text { Not } \\
\text { stated }\end{array}$ & 12.70 & (27) \\
\hline $\begin{array}{l}\text { Flanagan } \\
\text { et } a l^{\mathrm{d}}\end{array}$ & $\begin{array}{c}49.2^{\mathrm{e}} \\
(5.7-115)\end{array}$ & $\begin{array}{c}55 \\
(36-70)\end{array}$ & 18 & $\begin{array}{l}\text { At margin: } 14 \\
1.1-2 \mathrm{~mm}: 4\end{array}$ & $\begin{array}{l}\text { RT: } 1 \\
\text { HT: } 3\end{array}$ & None & - & 0 & (28) \\
\hline
\end{tabular}

${ }^{\mathrm{a}}$ The authors excluded patients with a previous history of breast cancer or a concurrent diagnosis of invasive cancer. ${ }^{\mathrm{b}}$ Details for margins not given: 'Recurrences had margins of $<1$ mm'; 'PLCIS alone cases. ${ }^{\mathrm{d}}$ The authors excluded 28 patients with concomitant breast cancer. Of the 23 remaining, 5 underwent mastectomy and have been removed from the analysis. ${ }^{e}$ Mean. N, number of patients; LRR, local recurrence rate; $\mathrm{Rx}$, therapy; CT, chemotherapy; RT, radiotherapy; CRT, chemo-radiotherapy; HT, hormone therapy.

to study the risk of local recurrence following excision. The median follow-up period was 55.6 months, 6 local recurrences were reported of which 4 were invasive carcinoma and 2 were PLCIS only. None of these patients received radiotherapy. Also of note is that of the 11 patients who received hormonal therapy, 3 developed local recurrences. Khoury et al (27) identified a statistically significant correlation between tumour recurrence and patient age in which the mean age for recurrence was 52.5 years compared to 60.6 years for those who did not develop recurrences (27).

The most recent study to comment on recurrence rates was that of Flanagan et al (28) who reported on a case series of 23 patients who were diagnosed with PLCIS. Of these 23 patients, 2 had adequate margins on the excisional biopsy and required no further intervention, while 16 underwent wide local excision and 5 had mastectomies. However even among the 16 who had WLE there was difficulty in getting clear margins and were subjected to re-excisions. Eventually another 7 patients required mastectomies as well. Over the 4 years of follow-up, the authors reported no instances of local recurrence. However, they also emphasize that the pursuit of clear margins in the case of PLCIS precluded successful breast conservation. Therefore, the authors urge caution in the aggressive treatment of PLCIS with regards to margins (28).

The retrospective nature of the data and the inconsistency in reporting of surgical margins preclude the formulation of an objective recommendation concerning the same. All of the authors appear to agree that clear margins will lead to lower recurrence rates. However, Flanagan et al (28) report that this may not always be possible or feasible.

Similarly, there is a lack of consistency in the adjuvant therapy given to patients, with little clarity in the literature concerning the criteria used to guide such decisions. It would be desirable to form a consensus with regards to the place of adjuvant therapy with regards to the treatment of PLCIS. As observed above, the numbers of patients in these studies are not sufficient to lead to any solid conclusions. However, Downs-Kelley et al (26) and Khoury et al (27) and indicated that radiotherapy led to a lower recurrence rate in their case series. Furthermore, Khoury et al (27) noted that patients on hormone therapy contributed to half of the recurrence cases. This issue may need to be explored in larger well-designed prospective studies.

\section{Conclusion}

The literature regarding PLCIS is relatively novel. In view of the rarity of this condition, usable evidence is accumulating slowly. Consequently, it may not always be possible to formulate recommendations based on the appropriate evidence. However, on the basis of the observed behaviour of the disease in retrospective studies, it would not be unreasonable to characterise PLCIS as significant risk factors for concomitant carcinoma with a significant risk of recurrence after excision. This is the position of the National Cancer Comprehensive Network (NCCN) and NHS breast screening program (NHSBSP) who recommend treating PLCIS similar to DCIS $(29,30)$. However, as evident in more recent studies, such an approach may not be consistent with breast conservation (28). This clinical issue warrants investigation in appropriately powered, well-designed, prospective studies for a satisfactory resolution of all concerns.

\section{Acknowledgements}

The present study was funded by the Breast Cancer Hope Foundation (London, UK). 


\section{References}

1. Frost AR, Tsangaris TN and Silverberg SG: Pleomorphic lobular carcinoma in situ. Pathology Case Reviews 1: 27-31, 1996.

2. Blair SL, Emerson DK, Kulkarni S, Hwang ES, Malcarne V and Ollila DW: Breast surgeon's survey: No consensus for surgical treatment of pleomorphic lobular carcinoma in situ. Breast J 19: $116-118,2013$.

3. Hussain M and Cunnick GH: Management of lobular carcinoma in-situ and atypical lobular hyperplasia of the breast-a review. Eur J Surg Oncol 37: 279-289, 2011.

4. Pieri A, Harvey J and Bundred N: Pleomorphic lobular carcinoma in situ of the breast: Can the evidence guide practice? World J Clin Oncol 5: 546-553, 2014.

5. Haagensen CD, Lane N, Lattes R and Bodian C: Lobular neoplasia (so-called lobular carcinoma in situ) of the breast. Cancer 42: 737-769, 1978

6. Ciocca RM, Li T, Freedman GM and Morrow M: Presence of lobular carcinoma in situ does not increase local recurrence in patients treated with breast-conserving therapy. Ann Surg Oncol 15: 2263-2271, 2008

7. Chuba PJ, Hamre MR, Yap J, Severson RK, Lucas D, Shamsa F and Aref A: Bilateral risk for subsequent breast cancer after lobular carcinoma-in-situ: Analysis of surveillance, epidemiology, and end results data. J Clin Oncol 23: 5534-5541, 2005

8. Aulmann S, Penzel R, Longerich T, Funke B, Schirmacher P and Sinn HP: Clonality of lobular carcinoma in situ (LCIS) and metachronous invasive breast cancer. Breast Cancer Res Treat 107: 331-335, 2008.

9. Bagaria SP, Shamonki J, Kinnaird M, Ray PS and Giuliano AE: The florid subtype of lobular carcinoma in situ: Marker or precursor for invasive lobular carcinoma? Ann Surg Oncol 18: $1845-1851,2011$

10. Lien HC, Chen YL, Juang YL and Jeng YM: Frequent alterations of HER 2 through mutation, amplification, or overexpression in pleomorphic lobular carcinoma of the breast. Breast Cancer Res Treat 150: 447-455, 2015.

11. Green AR, Young P, Krivinskas S, Rakha EA, Claire Paish E, Powe DG and Ellis IO: The expression of ERalpha, ERbeta and PR in lobular carcinoma in situ of the breast determined using laser microdissection and real-time PCR. Histopathology 54: 419-427, 2009

12. Sneige N, Wang J, Baker BA, Krishnamurthy $S$ and Middleton LP: Clinical, histopathologic, and biologic features of pleomorphic lobular (ductal-lobular) carcinoma in situ of the breast: A report of 24 cases. Mod Pathol 15: 1044-1050, 2002.

13. Chen YY, Hwang ES, Roy R, DeVries S, Anderson J, Wa C, Fitzgibbons PL, Jacobs TW, MacGrogan G, Peterse H, et al: Genetic and phenotypic characteristics of pleomorphic lobular carcinoma in situ of the breast. Am J Surg Pathol 33: 1683-1694, 2009.

14. Palacios J, Sarrio D, Garcia-Macias MC, Bryant B, Sobel ME and Merino MJ: Frequent E-cadherin gene inactivation by loss of heterozygosity in pleomorphic lobular carcinoma of the breast. Mod Pathol 16: 674-678, 2003.

15. Sapino A, Frigerio A, Peterse JL, Arisio R, Coluccia C and Bussolati G: Mammographically detected in situ lobular carcinomas of the breast. Virchows Arch 436: 421-430, 2000

16. Fadare O, Dadmanesh F, Alvarado-Cabrero I, Snyder R, Stephen Mitchell J, Tot T, Wang SA, Ghofrani M, Eusebi V, Martel M and Tavassoli FA: Lobular intraepithelial neoplasia [lobular carcinoma in situ] with comedo-type necrosis: A clinicopathologic study of 18 cases. Am J Surg Pathol 30: 1445-1453, 2006.
17. Carder PJ, Shaaban A, Alizadeh Y, Kumarasuwamy V, Liston JC and Sharma N: Screen-detected pleomorphic lobular carcinoma in situ (PLCIS): Risk of concurrent invasive malignancy following a core biopsy diagnosis. Histopathology 57: 472-478, 2010.

18. Chivukula M, Haynik DM, Brufsky A, Carter G and Dabbs DJ: Pleomorphic lobular carcinoma in situ (PLCIS) on breast core needle biopsies: Clinical significance and immunoprofile. Am J Surg Pathol 32: 1721-1726, 2008.

19. Fasola CE, Jensen KC and Horst KC: Local regional recurrence among patients with pleomorphic lobular carcinoma in situ: Is there a role for radiation therapy? International Journal of Radiation Oncology*Biology*Physics 84: S238, 2012.

20. Morris K, Howe M, Kirwan C and Harvey J: Clinical and phenotypic characteristics of core biopsy diagnosed pleomorphic lobular carcinoma-in-situ in a UK population (PLCIS). European Journal of Surgical Oncology (EJSO) 39: 484, 2013.

21. Niell B, Specht M, Gerade B and Rafferty E: Is excisional biopsy required after a breast core biopsy yields lobular neoplasia? AJR Am J Roentgenol 199: 929-935, 2012.

22. Lavoué V, Graesslin O, Classe JM, Fondrinier E, Angibeau H and Levêque J: Management of lobular neoplasia diagnosed by core needle biopsy: Study of 52 biopsies with follow-up surgical excision. Breast 16: 533-539, 2007.

23. Georgian-Smith D and Lawton TJ: Calcifications of lobular carcinoma in situ of the breast: Radiologic-pathologic correlation. AJR Am J Roentgenol 176: 1255-1259, 2001.

24. Mahoney MC, Robinson-Smith TM and Shaughnessy EA: Lobular neoplasia at 11-gauge vacuum-assisted stereotactic biopsy: Correlation with surgical excisional biopsy and mammographic follow-up. AJR Am J Roentgenol 187: 949-954, 2006.

25. Purdie CA, McLean D, Stormonth E, Macaskill EJ, McCullough JB, Edwards SL, Brown DC and Jordan LB: Management of in situ lobular neoplasia detected on needle core biopsy of breast. J Clin Pathol 63: 987-993, 2010.

26. Downs-Kelly E, Bell D, Perkins GH, Sneige N and Middleton LP: Clinical implications of margin involvement by pleomorphic lobular carcinoma in situ. Arch Pathol Lab Med 135: 737-743, 2011.

27. Khoury T, Karabakhtsian RG, Mattson D, Yan L, Syriac S, Habib F, Liu S and Desouki MM: Pleomorphic lobular carcinoma in situ of the breast: Clinicopathological review of 47 cases. Histopathology 64: 981-993, 2014.

28. Flanagan MR, Rendi MH, Calhoun KE, Anderson BO and Javid SH: Pleomorphic lobular carcinoma in situ: Radiologic-pathologic features and clinical management. Ann Surg Oncol 22: 4263-4269, 2015.

29. N.C.C.N. guidelines: Breast cancer. National Comprehensive Cancer Network, Fort Washington PA, USA, 2015. http:// www.ncen.org/patients/guidelines/stage_0_breast/index.html. Accessed, July 2015.

30. Guidelines for pathology reporting in breast disease. NHS Cancer Screening Programmes \& Royal College of Pathologists, South Sheffield, UK, 2005. http://www.cancerscreening.nhs.uk/ breastscreen/publications/nhsbsp58.html. Accessed, July 2015. 